\title{
Outbreak of Mycobacterium tuberculosis Beijing Strain in a High School in Yunnan, China
}

\author{
Jinglong Hou, ${ }^{1} \dagger$ Yu Pang, ${ }^{2} \dagger$ Xing Yang, ${ }^{1}$ Tao Chen, ${ }^{1}$ Huijuan Yang, ${ }^{1}$ Rui Yang, ${ }^{1}$ Lianyong Chen, ${ }^{1}$ and Lin Xu ${ }^{1 *}$ \\ ${ }^{1}$ Yunnan Provincial Center for Disease Control and Prevention, Kunming, China; ${ }^{2}$ Beijing Chest Hospital, Beijing Tuberculosis and Thoracic \\ Tumor Institute, Capital Medical University, Beijing, China
}

\begin{abstract}
In this study, we report an investigation of a tuberculosis (TB) outbreak in a high school in China. Eleven students with active TB were identified. A culture-negative 17-year-old girl was considered as index case affected by pulmonary and meningeal TB. Screening results indicated latent TB in $32.8 \%$ of the students in the classroom of index case, whereas a significantly decreased prevalence of TB infection was found among students on the same floor, ranging from 1.3 to $8.2 \%$. Genotyping revealed that all the Mycobacterium tuberculosis (MTB) isolates belonged to Beijing spoligotype international types 1 (SIT1). In conclusion, a diagnostic delay for the culture-negative index case played an important role in the transmission of Beijing genotype MTB strain in the boarding school in Yunnan. The separate locations of classrooms and sufficient air ventilation contributed to the significant difference in proportions of TB infection between classmates and other students in this outbreak.
\end{abstract}

Tuberculosis (TB), caused by Mycobacterium tuberculosis complex, remains a major public health problem globally. ${ }^{1,2}$ Despite showing a declining trend over the past decade, there were approximate 10.0 million new cases and 1.6 million deaths due to TB worldwide in $2017 .^{2}$ As an airborne disease, MTB infection is acquired by inhalation of infectious aerosol particles released from close contacts. ${ }^{3}$ Tuberculosis outbreaks thus mostly emerge in conditions of relative overcrowding, such as schools, prisons, and refugee camps. Although intermittent outbreaks of TB have limited impact on the national incidence rate in high-burden settings, they can put tremendous stress on local public health resources. ${ }^{4}$ Recognizing the transmission of pulmonary TB and characteristics of outbreak-related patients could help focus interventions to interrupt transmission in the community. ${ }^{5,6}$

China is a high-prevalence country for TB, with an estimated incidence of 63 cases $/ 100,000$ inhabitants in $2017 .^{2}$ Yunnan, located in the southwestern China, is a typical ethnic minority area, which has the similar incidence in pulmonary TB with the national level (56 cases $/ 100,000$ inhabitants). ${ }^{7}$ However, the marked diversity in TB prevalence is noted across various regions of Yunnan, majorly contributing to different levels of economic development. ${ }^{7}$ More importantly, the preferential boarding high schools are more frequently seen as a key strategy to improve the quality of education in the economically lagging regions. ${ }^{8}$ As a consequence, these two aspects synergistically increase the risk of emergence of TB outbreak in this region. In this study, we investigated a TB outbreak identified in 2018 among individuals in a boarding high school.

In March 2018, pulmonary and meningeal TB were identified in a 17-year-old girl in Yunnan who was in the second year of high school. The girl experienced cough in January 2018 and self-administered antibiotics. Two months later, the girl sought care in Yunnan Provincial Hospital of Infectious Diseases because of aggravated severity of symptoms. The second case was identified as smear-positive pulmonary TB 2 days after confirmation of index case. In addition, the third and fourth active pulmonary TB cases were reported in the next 2 weeks, respectively. All these cases were from the same class.

\footnotetext{
*Address correspondence to Lin Xu, Yunnan Provincial Center for Disease Control and Prevention, No. 158, Dongsi St., Kunming, China 650022. E-mail: 123316859@qq.com

$\dagger$ These authors contributed equally to this work.
}

Local health authorities conducted a contact investigation as a result of these four cases. Tuberculin skin tests (TSTs) were performed using $5 \mathrm{IU}$ of TB-PPD. The induration of $\geq 15 \mathrm{~mm}$ is considered positive according to the national guidelines for tuberculosis screening. Those with positive TST results undergo clinical evaluation and chest radiography. The student was defined as a case of latent TB infection (LTBI) after exclusion of active TB cases.

Epidemiological investigation was carried out during April and May 2018 and included all 395 students and staff whose classrooms were located in the same floor as that of index case. All of these individuals received TST. Screening results indicated LTBI for $32.8 \%$ of the students in the classroom of index case, whereas a significantly decreased ratio of TB infection was found among students on the same floor, ranging from 1.3 to $8.2 \%(P<0.01$, Figure $1 \mathrm{~A})$. When the nonclassmates were used as the control group, the classmates of index case were more likely to be infected with TB (odds ratio: 10.46, 95\% Cl: 4.92-22.23). In addition, five classmates of index case were clinically diagnosed as active pulmonary TB cases. Three sputum specimens were collected from each case for further laboratory examination, including smear microscopy and mycobacterial culture. After detection with multiple laboratory methods, five cases were identified as culture-positive cases, and the other two were clinically diagnosed TB cases (Table 1). In total, four isolates were collected in this study. Genotyping revealed that all the four isolates belonged to Beijing SIT1 spoligotype. Whole-genome sequencing of MTB isolates was conducted using a HiSeq 4000 system (lllumina, San Diego, CA). The MUMmer (http:// mummer.sourceforge.net/) and LASTZ (http://www.bx.psu.edu/ miller_lab/dist/README.lastz-1.02.00/) software were used to identify single-nucleotide polymorphisms and insertions/ deletions by comparison with the reference MTB H37Rv sequence (accession no. NC_000962), respectively. Our results confirmed that all the four isolates harbored the same genome sequence (accession on. PRJNA595750), suggesting all the four patients belonged to a single transmission chain (Supplemental Figure 1).

We further analyzed the distribution of active TB cases in the classroom and dormitory. As shown in Figure 1B, all these cases came from the same class, including 10 girls and one boy, who distributed dispersedly across classroom seats. Of these 11 cases, nine were resident students and the other two 


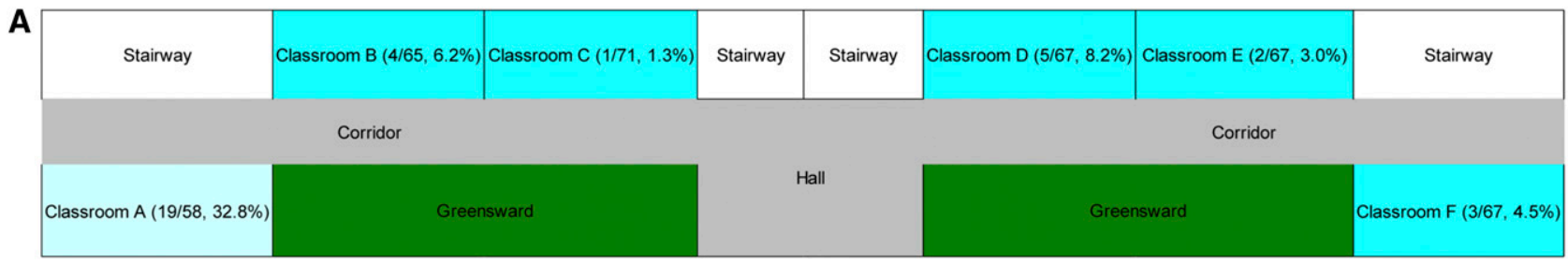

B
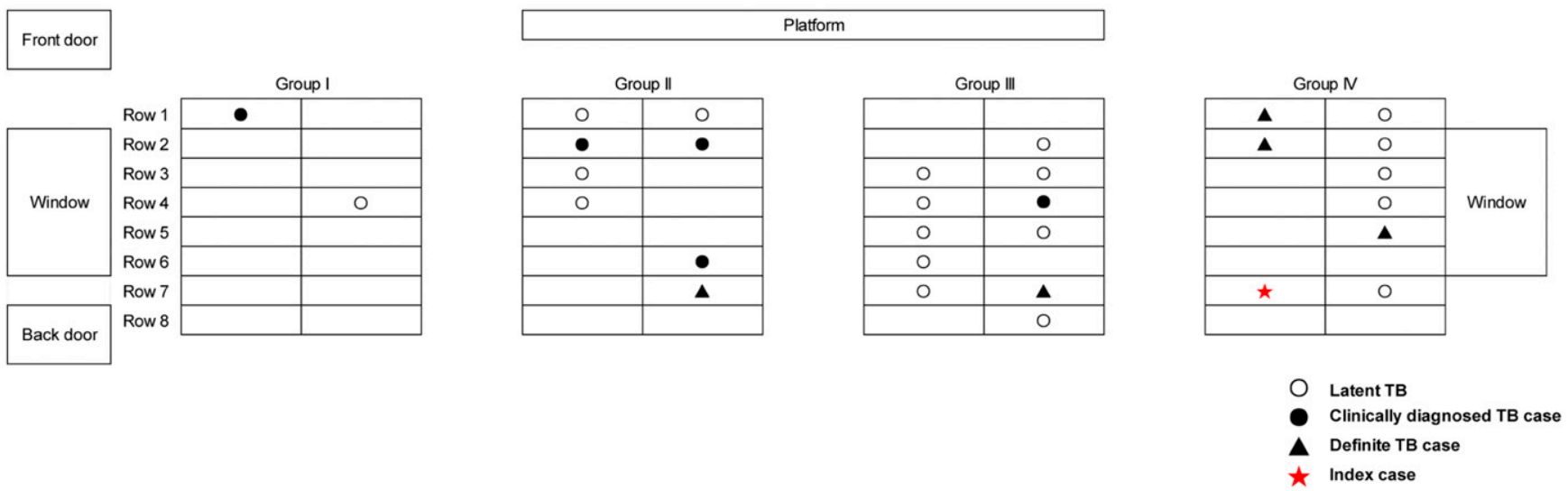

FIGURE 1. Distribution of active tuberculosis (TB) cases and TB infections in this investigation. (A) Floor plan of classrooms at high school in Yunnan. Values are number of TB infections/total number of students tested in classroom. Shading indicates classroom is that of index case. (B) Distribution of active TB cases and TB infections in the classroom where the index case lived.

were non-residant students. These resident students were located in six dormitories. Three cases emerged in the dormitory where the index case lived. During 1-year follow-up for the LTBI students and staff members in this school, none developed active TB cases.

We speculated that YN001 was the most probable source case in view of the earliest onset of TB symptoms. On the one hand, there is strong evidence that TB patients with smearnegative TB are responsible for about one-quarter of transmission in the community, ${ }^{9,10}$ indicating that the patients with low bacillary load could also contribute to the apparent transmissibility of TB. Given that this source case was culture negative by laboratory examinations, the potential transmission in this outbreak reflects that the concentration of tubercle bacilli in the specimens may fail to achieve the lowest detection limit of mycobacterial culture because of empirical treatment with fluoroquinolone. Thus, there is an urgent need for the development of promising diagnostics with increased sensitivity for these paucibacillary TB patients. ${ }^{11}$ Recently, GeneXpert Ultra has a limit of detection of approximately 16 colony-forming units (CFU/mL) compared with $114 \mathrm{CFU} / \mathrm{mL}$ for Xpert MTB/RIF (Cepheid, Sunnyvale, CA), which provide a promising alternative for early identification of index case. ${ }^{12}$ On the other hand, the potential transmission of smear-negative TB cases was attributed to a diagnose delay with empirical selftreatment. In other words, this 17-year-old patient may be "smear positive" before the time point she was diagnosed. Therefore, the diagnosis delay of index case plays an important role in accelerating the transmission in this school.

Another interesting finding of this study was that this outbreak was limited in contacts in the sole classroom. This result is consistent with our observation that the significant low rate

TABLE 1

Description of cases in a school tuberculosis outbreak, Yunnan, China

\begin{tabular}{|c|c|c|c|c|c|c|c|c|}
\hline \multirow{2}{*}{$\begin{array}{c}\text { ID of } \\
\text { patients }\end{array}$} & \multirow[b]{2}{*}{ Gender } & \multirow{2}{*}{$\begin{array}{c}\text { Age } \\
\text { (years) }\end{array}$} & \multirow[b]{2}{*}{ Date of onset of symptoms } & \multirow[b]{2}{*}{ Date of TB diagnosis } & \multicolumn{2}{|c|}{ Laboratory examination } & \multirow[b]{2}{*}{ Classification* } & \multirow[b]{2}{*}{ TB infection site } \\
\hline & & & & & Smear microscopy & Culture & & \\
\hline YN01 & Female & 17 & January 1, 2018 & March 14, 2018 & - & - & Clinically diagnosed TB & Lung, meningitis \\
\hline YN02 & Female & 17 & December 1, 2018 & March 16, 2018 & + & + & Definitive TB & Lung \\
\hline YN03 & Female & 17 & January 20, 2018 & March 23, 2018 & - & - & Clinically diagnosed TB & Lung \\
\hline YN04 & Female & 16 & February 21, 2018 & March 27, 2018 & - & - & Clinically diagnosed TB & Lung, pleurisy \\
\hline YN05 & Female & 16 & March 1, 2018 & April 5, 2018 & - & + & Definitive TB & Lung \\
\hline YN06 & Female & 17 & March 10, 2018 & April 5, 2018 & + & + & Definitive TB & Lung \\
\hline YN07 & Male & 17 & March 19, 2018 & April 5, 2018 & + & + & Definitive TB & Lung, pleurisy \\
\hline YN08 & Female & 16 & March 31, 2018 & April 5, 2018 & - & + & Definitive TB & Lung \\
\hline YN09 & Female & 17 & April 1, 2018 & April 5, 2018 & + & - & Definitive TB & Lung \\
\hline YN10 & Female & 17 & April 12, 2018 & April 20, 2018 & - & - & Clinically diagnosed TB & Lung \\
\hline YN11 & Female & 18 & April 12, 2018 & April 21, 2018 & & - & Clinically diagnosed TB & Lung \\
\hline
\end{tabular}

$\mathrm{TB}=$ tuberculosis.

* Definitive TB was defined as the case with clinical symptoms plus at least one sputum sample smear positive and/or culture positive for MTB; clinically diagnosed TB was defined as the case with clinical TB symptoms plus favorable response to anti-TB therapy, but without any sputum sample smear positive or culture positive for MTB. 
of LTBI was identified for the students from the neighboring classrooms, and no new case emerged over the 1-year followup period. This phenomenon could be explained by building designs of this school. On the one hand, luckily, the classroom where this outbreak emerged was located in the corner of the teaching building, and the spatial separation between this classroom and others effectively prevents the TB transmission. On the other hand, room doors of both classroom and dormitory open onto the outdoor corridors. As a consequence, the sufficient air ventilation is responsible for the reduction in the aerosol concentration with tubercle bacilli. Our data confirm that the enhanced natural ventilation is an effective measure to prevent the transmission of airborne infectious diseases, such as TB.

There were several obvious limitations in our study. On the one hand, although the frequent contact is an important variable affecting the TB infections during the outbreak, the data on this aspect were not collected in this study. On the other hand, the small sample number of active cases limits the potential for analysis of individual risk of TB patients among the school population. Future study will be carried out on this aspect as this will provide the high-risk groups of infection for further investigation and prevention.

The results of this investigation indicate that a diagnostic delay of culture-negative index case plays an important role in the transmission of Beijing genotype MTB strain in the boarding school in Yunnan. The separate location of classroom and sufficient air ventilation majorly contribute to the significant difference in proportions of TB infection between classmates and non-classmates in this outbreak.

Received July 18, 2019. Accepted for publication December 29, 2019.

Published online February 24, 2020.

Note: Supplemental figure appears at www.ajtmh.org.

Acknowledgment: We thank the staff taking part in this study for their hard work.

Authors' addresses: Jinglong Hou, Xing Yang, Tao Chen, Huijuan Yang, Rui Yang, Lianyong Chen, and Lin Xu, Yunnan Provincial Center for Disease Control and Prevention, Kunming, China, E-mails: 979977862@qq.com, yangxing27@126.com,946579325@qq.com, 237535956@qq.com, 891873799@qq.com, ynchenly@163.com, and 123316859@qq.com. Yu Pang, Beijing Chest Hospital, Beijing Tuberculosis and Thoracic Tumor Institute, Capital Medical University, Beijing, China, E-mail: pangyupound@163.com.

\section{REFERENCES}

1. Wang $L$ et al., 2014. Tuberculosis prevalence in China, 1990-2010; a longitudinal analysis of national survey data. Lancet 383: 2057-2064.

2. World Health Organization, 2018. Global Tuberculosis Report 2018. WHO/CDS/TB/2018.20. Geneva, Switzerland: WHO.

3. Fennelly KP et al., 2012. Variability of infectious aerosols produced during coughing by patients with pulmonary tuberculosis. Am J Respir Crit Care Med 186: 450-457.

4. Mitruka K, Oeltmann JE, ljaz K, Haddad MB, 2011. Tuberculosis outbreak investigations in the United States, 2002-2008. Emerg Infect Dis 17: 425-431.

5. Haddad MB, Mitruka K, Oeltmann JE, Johns EB, Navin TR, 2015. Characteristics of tuberculosis cases that started outbreaks in the United States, 2002-2011. Emerg Infect Dis 21: 508-510.

6. Faccini $\mathrm{M}$ et al., 2013. Tuberculosis outbreak in a primary school, Milan, Italy. Emerg Infect Dis 19: 485-487.

7. Chen L, Pang Y, Ma L, Yang H, Ru H, Yang X, Yan S, Jia M, Xu L, 2017. First insight into the molecular epidemiology of $M y c O-$ bacterium tuberculosis isolates from the minority enclaves of southwestern China. Biomed Res Int 2017: 2505172.

8. Stein-Zamir C, Volovik I, Rishpon S, Atamna A, Lavy A, WeilerRavell D, 2006. Tuberculosis outbreak among students in a boarding school. Eur Respir J 28: 986-991.

9. Tostmann A, Kik SV, Kalisvaart NA, Sebek MM, Verver S, Boeree MJ, van Soolingen D, 2008. Tuberculosis transmission by patients with smear-negative pulmonary tuberculosis in a large cohort in The Netherlands. Clin Infect Dis 47: 1135-1142.

10. Behr MA, Warren SA, Salamon H, Hopewell PC, Ponce de Leon A, Daley CL, Small PM, 1999. Transmission of Mycobacterium tuberculosis from patients smear-negative for acid-fast bacilli. Lancet 353: 444-449.

11. Parsons LM, Somoskovi A, Gutierrez C, Lee E, Paramasivan CN, Abimiku A, Spector S, Roscigno G, Nkengasong J, 2011. Laboratory diagnosis of tuberculosis in resource-poor countries: challenges and opportunities. Clin Microbiol Rev 24: 314-350.

12. World Health Organization, 2017. WHO Meeting Report of a Technical Expert Consultation: Non-inferiority Analysis of Xpert MTF/RIF UItra Compared to Xpert MTB/RIF. Geneva, Switzerland: World Health Organization (WHO/HTM/TB/2017.04). 